\title{
Serum levels of matrix metalloproteinases MMP-2, MMP-3, MMP-9 and tissue inhibitor of matrix metalloproteinase TIMP-2 in patients with myocarditis.
}

\section{Małgorzata Kobusiak-Prokopowicz ( $\square$ kobusiak@poczta.fm )}

Wroclaw Medical University: Uniwersytet Medyczny im Piastow Slaskich we Wroclawiu https://orcid.org/0000-0001-5718-5702

\section{Konrad Kaaz}

Wroclaw Medical University: Uniwersytet Medyczny im Piastow Slaskich we Wroclawiu

\section{Dominik Marciniak}

Wroclaw Medical University: Uniwersytet Medyczny im Piastow Slaskich we Wroclawiu

\section{Bożena Karolko}

Wroclaw Medical University: Uniwersytet Medyczny im Piastow Slaskich we Wroclawiu Andrzej Mysiak

Wroclaw Medical University: Uniwersytet Medyczny im Piastow Slaskich we Wroclawiu

\section{Research}

Keywords: chronic kidney disease, matrix metalloproteinase, myocarditis, extracellular matrix, tissue inhibitor of metalloproteinases

Posted Date: March 29th, 2021

DOI: https://doi.org/10.21203/rs.3.rs-307916/v1

License: (9) This work is licensed under a Creative Commons Attribution 4.0 International License. Read Full License 


\section{Abstract}

Background: Under physiological conditions, the myocardial extracellular matrix (ECM) is maintained by matrix metalloproteinases (MMPs) and tissue inhibitors of metalloproteinases (TIMPs). However, certain stimuli cause the upregulation of MMPs, which can lead to pathological remodeling of the ECM. We assessed serum levels of MMPs and TIMP-2 in patients with myocarditis and their relationship(s) to myocardial damage.

Methods: In total, 45 patients with myocarditis who underwent cardiac magnetic resonance imaging were included, comprising 11 with concurrent chronic kidney disease (CKD). Blood samples were obtained to assess serum levels of MMP-2, MMP-3, MMP-9, and TIMP-2.

Results: Serum MMP-2, MMP-3, and TIMP-2 levels negatively correlated with ejection fraction values in patients with myocarditis, while MMP-3 levels correlated with longitudinal deformation $(p<0.05)$. Serum MMP-2, MMP-3, and TIMP-2 levels also negatively correlated with renal function, as assessed by the estimated glomerular filtration rate $(p<0.05)$. Patients with myocarditis and concurrent CKD had higher levels of MMP-2 and TIMP-2 than those without kidney damage.

\section{Conclusions:}

1. We demonstrated serum MMP-2, MMP-3, and TIMP-2 concentrations were related to left ventricular ejection fraction, and MMP-3 levels correlated with longitudinal deformation, indicating MMPs play an important role in the post-inflammatory remodeling of the myocardium.

2. The occurrence of other heart diseases was an important element in modifying the relationship between MMPs and the degree of myocardial damage.

3. Chronic kidney damage in patients with myocarditis results in increased MMP activity. A negative correlation between eGFR and MMP-2, MMP-3 and TIMP-2, and a positive correlation between creatinine and MMP-3 levels, underlines the role of fibrosis in myocarditis with concomitant chronic kidney disease.

\section{Introduction}

The extracellular matrix (ECM) protects the geometry and integrity of myocardial tissues by creating a "skeleton" upon which matrix proteins can anchor, and maintaining homeostasis between protein deposition and degradation [1,2]. ECM transformations affect various physiological processes, including cell growth and differentiation, as well as tissue development and formation [3]. The ECM is also responsible for the transmission of mechanical forces within the vessels and heart, and affects the diastolic susceptibility of the arteries [4].

The main mediators of remodeling within the ECM are matrix metalloproteinases (MMPs), i.e., enzymes with the ability to degrade structural matrix proteins. Although the production of MMPs is usually low in most tissues, certain stimuli (e.g. tissue damage, hyperglycemia, proinflammatory factors, and 
aldosterone) can upregulate their transcription [5,6]. Under physiological states, ECM remodeling by MMPs is strictly controlled by endogenous tissue inhibitors of metalloproteinases (TIMPs). However, changes in the balance between MMPs and TIMPs have been shown to be involved in pathological remodeling of the heart muscle and can contribute to acute damage during ischemia [1,2,7]. Indeed, there is a general tendency to increase the proteolytic activity of MMPs in the myocardium during ischemia [7]. Such irreversible ECM remodeling has been shown to be responsible for both compensatory hypertrophy and decompensated congestive heart failure [8]. Furthermore, the ventricular muscle remodeling following myocardial infarction or damage due to viral infections has also been shown to be mediated by MMPs $[8,9]$.

MMPs can be divided into five groups based on their substrate and structure: matrylisins, collagenases, stromelysins, gelatinases, and membrane metalloproteinases. Among them, MMP-2 and MMP-9 (also called gelatinases $A$ and $B$ ), which both digest structural proteins of the collagen-elastin network, are implicated in the pathogenesis of coronary syndromes and heart failure [10-12]. Elevated MMP-2 levels have been shown to stimulate adverse vascular remodeling and thrombotic effects, which accelerate vascular damage $[6,13]$. Increased MMP-2 and MMP-9 activity have also been shown to be associated with persistent low-grade inflammation [14] and endothelial dysfunction [15]. Therefore, MMPs may contribute to the myocardial changes observed in myocarditis.

Elevated MMP levels may also have significant effects on the kidney. Indeed, increased MMP-9 levels have been shown to be associated with kidney damage [16]. Increased MMP-3 (a stromelysin MMP) levels may also cause kidney damage, as one of the main MMP-3 substrates is type IV collagen, an important component of the basal membranes of the renal tubules and vessels [17]. MMP-2 has also been shown to increase fibrosis in the kidney [18]. Therefore, serum MMP levels may also influence kidney function in patients with myocarditis.

As serum concentrations of MMPs typically increase as TIMP levels decrease, changes in TIMP levels may also occur in cardiovascular disease. Indeed, such an imbalance in favor of proteinases is associated with increased transformation and cardiovascular disease progression [5]. There are four types of TIMPS (TIMP-1-4). However, different TIMPs inhibit the various MMPs better than others; for example, TIMP-2 inhibits MMP-2 more effectively than other TIMPs. Therefore, reduced levels of TIMP-2, in particular, may occur in myocarditis.

\section{Study objective}

The aim of the study was to assess the activity of MMPs, as well as the balance between MMPs and TIMP-2, in the serum of patients with myocarditis as elements contributing to the remodeling of the ECM and heart damage.

\section{Material And Methods}


Fifty-eight patients hospitalized in the Cardiology Department from 2013 to 2017 with myocarditis diagnosed based on the European Society of Cardiology (ESC) recommendations [19] were qualified for the study. Patients underwent standard laboratory tests, electrocardiography, and echocardiography (GE Vivid E9) during the first 24 hours of hospitalization. Each enrolled patient had a venous blood sample drawn from an antecubital vein within 24 hours of admission, to assess the serum levels of MMP-2, MMP-3, MMP-9, and TIMP-2. Blood samples were collected using a closed blood sampling system (BD Vacutainer ${ }^{\circledR}$ ); $4 \mathrm{ml}$ of blood was drawn into a tube containing EDTA (for plasma recovery) and $4 \mathrm{ml}$ into a clot activator serum tube (for serum recovery). After centrifugation and fractionation, the separated serum and plasma samples were stored at $-72^{\circ} \mathrm{C}$ until analysis. MMP-2, MMP-3, MMP-9, and TIMP-2 concentrations were determined in the preserved blood serum using R\&D Systems Quantikine ${ }^{\circ}$ ELISA kits (Bio Techne). Within 30 days of admission, cardiac magnetic resonance imaging (MRI) was performed in patients using a General Electric Signa HDxT 1.5T scanner.

For the purposes of the presented study, patients with at least grade 2 hypertension, heart failure, ischemic heart disease, or at least moderate valve disease were included in the group with significant heart disease prior to hospitalization.

Renal function was expressed as the estimated glomerular filtration rate (eGFR). eGFR was calculated by the abbreviated Modification of Diet in Renal Disease (MDRD) formula:

eGFR $=186 \times \mathrm{SCr}-1.154 \times($ age $)-0.203 \times(0.742$ if female $)$

where: eGFR indicates estimated GFR $\left(\mathrm{ml} / \mathrm{min} / 1.73 \mathrm{~m}^{2}\right)$ and $\mathrm{SCr}$ is the serum creatinine level.

The following parameters were assessed by echocardiography: left ventricular ejection fraction (LVEF), left ventricular end-diastolic dimension (LVEDD), left ventricular end-systolic dimension (LVESD), global longitudinal strain (GLS), and segmental contractility disorders. Echocardiography was performed in all patients within 48 hours of admission using a General Electric Vingmed Echocardiography system with a 2.5 $\mathrm{MHz}$ phased-array transducer. LVEF was calculated using the universal formula: EF = (LVEDV LVESV)/LVEDV $\times 100 \%$, where LVEDV indicates left ventricular end-diastolic volume and LVESV is the left ventricular end-systolic volume. LVEDV and LVESV were calculated using the Simpson biplane method, in which the machine computes the left ventricular volume based on perpendicular cross-sections of the left ventricle on the apical two- and four-chamber view, as determined by the operator.

Coronary angiography was performed in 29 patients, showing epicardial arterial changes in two of them. Cardiac MRI was also used to assess LVEF, LVEDD, LVESD, and myocarditis-specific features, including swelling and late gadolinium enhancement (LGE) using a General Electric Signa HDxT 1.5Tscanner. The final analysis included 45 patients (7 women, 38 men), comprising 11 with chronic kidney disease, who had cardiac MRI. Thirteen patients did not have an examination due to prior diagnosis, contraindications (presence of magnetic material), disagreement or lack of cooperation of the patient. Among patients undergoing cardiac MRI, three did not have contrast during the study, and LGE was confirmed in the subepicardial layer indicating myocarditis in 25 patients. In addition, cardiomyopathy was diagnosed in 
three cases: one case each of ischemic, hypertrophic, and dilated cardiomyopathy. The detailed characteristics of the studied group are presented in Table 1.

\section{Statistical methods}

The analyzed variables were both of nominal nature, including dichotomous and interval variables. Basic descriptive statistics were determined for interval variables: mean, standard deviation (SD), minimum and maximum values, and a $95 \%$ confidence interval $(\mathrm{Cl})$.

Contingency tables were the basis of the statistical analysis of variables in nominal scales, and Pearson's linear correlation matrices for interval variables. The statistical significance of the determined correlation coefficients $r$ was verified by the $t$ test, with the assumed confidence level $a=0.05$. First, the general relationships between the analyzed variables were assessed using principal component analysis (PCA). The PCA model was estimated using the NIPALS iterative algorithm. The convergence criterion was set at 0.00001 , setting the maximum number of iterations equal to 50 . The number of components was defined by determining the maximum predictive relevance by $\mathrm{V}$-fold cross-validation, setting their maximum number at $\mathrm{V}_{\max }=7$.

The resulting optimal PCA model was finally reduced to two components. The results of the PCA analysis are presented in Tables 2 and 3. The loading plot $p 1$ vs. $p 2$ allowed us to pre-determine the variables that have the most significant impact on the built model and select the most significant correlations between them. The variables selected in this way were then subjected to further statistical evaluation. Based on the results of the PCA, the nominal dichotomous variable Significant heart disease (YES/NO) was considered the basic grouping variable. To determine its correlation with other variables, univariate logistic regression was used with the free term $\neq 0$. The parameters of the logit function were estimated by the method of least squares using the Quasi-Newton algorithm at the initial default parameters values of 0.1 , setting the initial step size equal to 0.01 . The convergence criterion for the logistic regression was set at 0.00001 . To assess the degree of correlation of the independent variables with the dichotomous dependent variable, we used the logistic regression model to calculate the odds ratios (ORs) and their $95 \%$ Cls. The statistical significance of built logistic models was assessed by the chi-square test for degrees of freedom $d f=1$ and an assumed level of significance $(a)$ of 0.05 . Statistical analyzes were performed using the STATISTICA PL ${ }^{\circledR}$ version 13 software.

\section{Results}

Using PCA, we determined the most important parameter differentiating the study group is the history of significant heart disease (Table 2). Univariate logistic regression analysis for significant heart disease showed a significant increased OR for numerous parameters (Table 3 ).

Patients with chronic kidney damage had higher levels of MMP-2 and TIMP-2, although they were not significantly different from those with healthy kidneys in terms of $\mathrm{N}$-terminal pro B-type natriuretic peptide (NT-proBNP) levels and LVEF. 


\section{Correlations}

We observed negative correlations between eGFR and MMP-2, eGFR and MMP-3, and eGFR and TIMP-2 (Table 4). We also found creatinine positively correlated with MMP-3 concentration (Table 4). In addition, the correlation between MMP-2 and TIMP-2 levels in patients with chronic kidney damage did not differ significantly from those without.

We found a statistically significant negative correlation between MMP-2, MMP-3 and TIMP-2 concentration with LVEF (Table 5). Meanwhile, MMP-2, MMP-3 and TIMP-2 levels positively correlated with LVESD. MMP-3 values also correlated positively with GLS. TIMP-2 values correlated positively with LVEDD, GLS, and the LVESD assessed by MRI; and correlated negatively with the LVEF assessed by MRI (Table 5). There were no significant correlations between MMP-2, MMP-3, MMP-9, and TIMP-2 with serum C-reactive protein (CRP).

\section{Discussion}

Available data indicate extensive inflammatory processes exacerbate structural changes and may accelerate the development of heart failure [20-22]. In particular, increased collagen synthesis in the myocardium, along with its impaired degradation in response to pressure and volume overload, leads to collagen accumulation within the heart, causing structural remodeling, myocardial stiffness, and fibrosis $[23,24]$. Indeed, biomarkers of myocardial inflammation and ECM remodeling, such as MMPs, have previously been shown to be important predictors of mortality [25].

Here we found serum MMP-2, MMP-3, and TIMP-2 levels were significantly negatively correlated with ejection fraction values in patients with myocarditis. Elevated MMP-2 activity may lead to abnormal vascular remodeling by increasing the migration of vascular wall smooth muscle cells into the intima, increasing fibrosis, and reducing the elastin content $[6,26]$. In addition, MMP-2 increases the activity, adhesion, and aggregation of platelets and thus contributes to thrombus formation [13]. Furthermore, in patients with myocardial infarction, a higher initial concentration of MMP-2 was associated with a larger infarct area and a reduced ejection fraction in an observation lasting over several months [27]. Therefore, our results showing elevated serum MMP-2 levels and reduced cardiac function confirm the relationship already observed in patients with myocardial infarction.

We also observed a negative correlation between eGFR and MMP-2 levels. A previous study showed higher MMP-2 levels were associated with episodes of cardiovascular disease in patients with type 1 diabetes observed for 12 years, and that baseline eGFR weakened the relationship between MMP-2 and cardiovascular disease [28]. Therefore, the eGFR may at least partially shape the relationship between MMPs and cardiovascular diseases. This hypothesis is supported by the observation that MMP-2 causes changes that are characteristic for tubular epithelial-to-mesenchymal transition (EMT) of the kidneys, leading to their increased fibrosis, which, in turn, leads to impaired renal function [29]. Alternatively, higher MMP-2 levels may result from reduced kidney function, although this is less likely as serum MMP-2 levels 
are only slightly dependent on renal clearance (the molecular weight of MMP-2 is higher than that of albumin).

In addition to the negative correlation between eGFR and MMP-2, we observed a negative correlation between eGFR and MMP-3. MMP-3 concentration was also previously shown to be associated with a decrease in eGFR; however, the relationship between MMP-3 and total mortality did not change significantly after adjustment to the decrease in eGFR [30]. These results indicate increased concentrations of MMP-2 and -3 probably only partially contribute to the decrease in eGFR, which correlates positively with cardiovascular disease and total mortality [30].

MMPs may be of particular concern in heart failure patients with concurrent chronic kidney disease (CKD). Indeed, Du et al. showed MMP-2 and MMP-9 levels increased over time in damaged tubules and lead to renal fibrosis, which is a typical phenomenon in progressive renal disease [31]. Other studies have shown MMP-2 plays a pathological role in interstitial renal fibrosis, probably by inducing EMT and macrophage infiltration [32]. In addition, Neal et al. showed increased MMP-2, MMP-9, and TIMP-1 activity in the aorta of patients with progressive kidney disease, and increased activity of MMP-2 in the serum [33]. Increased tissue activity of MMP-2, MMP-9, and TIMP-2 levels were also demonstrated in rats with CKD, with elevated TIMP-2 levels likely compensating for the increased MMP activity. There was also a strong relationship between MMP-3 levels and albuminuria in rats with CKD, presumably due to increased MMP-3-dependent proteolysis of type IV collagen [17]. Furthermore, parallel studies in patients with CKD showed increased MMP-2 activity in arterial blood samples of patients who underwent kidney transplantation accompanied by vascular calcification [29].

The Hsu T-W et al. study demonstrated that baseline MMP-2, -3 and -9 levels were the independent predictors for faster eGFR decline and subsequent kidney disease progression. Their statistical analysis showed also, that low basal eGFR and higher MMP-9 levels were the independent predictors of mortality in CAD patients [34].

In our study, patients with chronic kidney damage had higher levels of MMP-2 and TIMP-2, although they were not significantly different from those with healthy kidneys in terms of N-terminal pro B-type natriuretic peptide (NT-proBNP) levels and LVEF. In addition, the correlation between MMP-2 and TIMP-2 levels in patients with chronic kidney damage did not differ significantly from those without, indicating the balance is maintained between the MMP and its inhibitor in those with kidney damage. Although such activation of MMP activity has already been observed in the cardiopulmonary setting and in severe inflammatory processes, our study is one of the first to show that chronic kidney damage in patients with myocarditis increases MMP activity. Therefore, inhibition of MMPs may be a useful therapeutic strategy in patients with myocarditis and concomitant CKD.

\section{Limitations}

There are several limitations to our study. First, plasma levels of MMP-2, $-3,-9$, and of TIMP-2 were only determined at baseline. Therefore, the changes in plasma levels over time, revealing more details about 
outcome measures, are not known. Second, we do not know to what extent plasma levels reflect the local pathological situation at the tissue level. Finally, we only assessed a few MMPs and only one of the four known TIMPs, so other MMPs and TIMPs may be involved in this pathogenic process.

\section{Conclusions}

1. We demonstrated serum MMP-2, MMP-3, and TIMP-2 concentrations were related to left ventricular ejection fraction, and MMP-3 levels correlated with longitudinal deformation, indicating MMPs play an important role in the post-inflammatory remodeling of the myocardium.

2. The occurrence of other heart diseases was an important element in modifying the relationship between MMPs and the degree of myocardial damage.

3. Chronic kidney damage in patients with myocarditis results in increased MMP activity.

4. A negative correlation between eGFR and MMP-2, MMP-3 and TIMP-2, and a positive correlation between creatinine and MMP-3 levels, underlines the role of fibrosis in myocarditis with concomitant chronic kidney disease.

\section{Declarations}

\section{Ethics approval}

This study was conducted in accordance with the Helsinki Declaration. The study design was accepted by the Bioethics Committee of the Wroclaw Medical University.

\section{Consent to participate}

Informed consent was obtained from each individual participant included in the study.

\section{Consent for publication}

We confirm that the manuscript has been read and approved by all named authors and that there are no other persons who satisfied the criteria for authorship but are not listed. We further confirm that the order of authors listed in the manuscript has been approved by all of us.

\section{Availability of data and material}

The data that support the findings of this study are available from the corresponding author upon reasonable request.

\section{Conflicts of interest/Competing interests}


The authors have no conflicts of interest that are relevant to the content of this article. The authors have no relevant financial or non-financial interests to disclose.

\section{Funding}

Uniwersytet Medyczny im. Piastów Slaskich we Wroclawiu Grant ST-908/ST.C150.15.068

\section{Authors' contributions}

All authors contributed to the study conception and design. Material preparation, data collection and analysis were performed by Małgorzata Kobusiak-Prokopowicz, Konrad Kaaz, Dominik Marciniak, Bożena Karolko and Andrzej Mysiak. The first draft of the manuscript was written by Małgorzata KobusiakProkopowicz and Konrad Kaaz and all authors commented on previous versions of the manuscript. All authors read and approved the final manuscript.

\section{Acknowledgements}

The authors thank Proper Medical Writing Sp. z o.o. for the language assistance provided in the preparation of this paper.

\section{References}

1. Graham HK, Horn M, Trafford AW. Extracellular matrix profiles in the progression to heart failure. European Young Physiologists Symposium Keynote Lecture-Bratislava 2007. Acta Physiol (Oxf). 2008 Sep; 194(1):3-21. doi: 10.1111/j.1748-1716.2008.01881.x.

2. Wilson EM, Spinale FG. Myocardial remodelling and matrix metalloproteinases in heart failure: turmoil within the interstitium. Ann Med. 2001 Dec;33(9):623-34.

3. Woessner JF Jr. Matrix metalloproteinases and their inhibitors in connective tissue remodeling. FASEB J. 1991 May;5(8):2145-54.

4. MacColl E, Khalil RA. Matrix Metalloproteinases as Regulators of Vein Structure and Function: Implications in Chronic Venous Disease. J Pharmacol Exp Ther. 2015 Dec;355(3):410-28. doi:10.1124/jpet.115.227330.

5. Fingleton B. Matrix metalloproteinases as valid clinical targets. Curr Pharm Des. 2007;13(3):333-46.

6. Chen Q, Jin M, Yang F, Zhu J, Xiao Q, Zhang L. Matrix metalloproteinases: inflammatory regulators of cell behaviors in vascular formation and remodeling. Mediators Inflamm. 2013;2013:928315. doi:10.1155/2013/928315.

7. Schulze CJ, Wang W, Suarez-Pinzon WL, Sawicka J, Sawicki G, Schulz R. Imbalance between tissue inhibitor of metalloproteinase-4 and matrix metalloproteinases during acute myocardial [correction of myocardial] ischemia-reperfusion injury. Circulation. 2003 May;20(19):2487-92. 107(.

8. Hendry RG, Bilawchuk LM, Marchant DJ. Targeting matrix metalloproteinase activity and expression for the treatment of viral myocarditis. J Cardiovasc Transl Res. 2014 Mar;7(2):212-25. doi: 
10.1007/s12265-013-9528-2.

9. Westermann D, Lindner D, Kasner M, Zietsch C, Savvatis K, Escher F, von Schlippenbach J, Skurk C, Steendijk P, Riad A, Poller W, Schultheiss HP, Tschöpe C. Cardiac inflammation contributes to changes in the extracellular matrix in patients with heart failure and normal ejection fraction. Circ Heart Fail. 2011 Jan;4(1):44-52. doi:10.1161/CIRCHEARTFAILURE.109.931451.

10. Creemers EE, Cleutjens JP, Smits JF, Daemen MJ. Matrix metalloproteinase inhibition after myocardial infarction: a new approach to prevent heart failure? Circ Res. 2001 Aug 3;89(3):201 - 10.

11. Jefferis BJ, Whincup P, Welsh P, Wannamethee G, Rumley A, Lennon L, Thomson A, Lawlor D, Carson C, Ebrahim S, Lowe G. Prospective study of matrix metalloproteinase-9 and risk of myocardial infarction and stroke in older men and women. Atherosclerosis. 2010 Feb;208(2):557-63. doi:10.1016/j.atherosclerosis.2009.08.018.

12. Spinale FG. Matrix metalloproteinases: regulation and dysregulation in the failing heart. Circ Res. 2002 Mar 22;90(5):520-30.

13. Gresele P, Falcinelli E, Loffredo F, Cimmino G, Corazzi T, Forte L, Guglielmini G, Momi S, Golino P. Platelets release matrix metalloproteinase-2 in the coronary circulation of patients with acute coronary syndromes: possible role in sustained platelet activation. Eur Heart J. 2011 Feb;32(3):31625. doi:10.1093/eurheartj/ehq390.

14. Chase AJ, Newby AC. Regulation of matrix metalloproteinase (matrixin) genes in blood vessels: a multi-step recruitment model for pathological remodelling. J Vasc Res. 2003 Jul-Aug;40(4):329-43.

15. Giebel SJ, Menicucci G, McGuire PG, Das A. Matrix metalloproteinases in early diabetic retinopathy and their role in alteration of the blood-retinal barrier. Lab Invest. 2005 May;85(5):597-607.

16. Ebihara I, Nakamura T, Shimada N, Koide H. Increased plasma metalloproteinase-9 concentrations precede development of microalbuminuria in non-insulin-dependent diabetes mellitus. Am J Kidney Dis. 1998 Oct;32(4):544-50.

17. Hosoyamada Y, Sakai T. Structural arrangement of collagen fibrils in the periarterial connective tissue of the kidney: their functional relevance as a structural stabilizer against arterial pressure. Anat Sci Int. 2012 Jun;87(2):80-7. doi:10.1007/s12565-011-0123-9.

18. Cheng S, Lovett DH. Gelatinase A (MMP-2) is necessary and sufficient for renal tubular cell epithelialmesenchymal transformation. Am J Pathol. 2003 Jun;162(6):1937-49. doi:10.1016/S00029440(10)64327-1.

19. Caforio AL, Pankuweit S, Arbustini E, Basso C, Gimeno-Blanes J, Felix SB, Fu M, Heliö T, Heymans S, Jahns R, Klingel K, Linhart A, Maisch B, McKenna W, Mogensen J, Pinto YM, Ristic A, Schultheiss HP, Seggewiss H, Tavazzi L, Thiene G, Yilmaz A, Charron P, Elliott PM, European Society of Cardiology Working Group on Myocardial and Pericardial Diseases. Current state of knowledge on aetiology, diagnosis, management, and therapy of myocarditis: a position statement of the European Society of Cardiology Working Group on Myocardial and Pericardial Diseases. Eur Heart J. 2013 Sep;34(33):2636-48. . doi: 10.1093/eurheartj/eht210. 
20. Boulogne M, Sadoune M, Launay JM, Baudet M, Cohen-Solal A, Logeart D. Inflammation versus mechanical stretch biomarkers over time in acutely decompensated heart failure with reduced ejection fraction. Int J Cardiol. 2017 Jan 1;226:53-59. doi: 10.1016/j.ijcard.2016.10.038.

21. AbouEzzeddine OF, McKie PM, Scott CG, Rodeheffer RJ, Chen HH, Michael Felker G, Jaffe AS, Burnett JC, Redfield MM. Biomarker-based risk prediction in the community. Eur J Heart Fail. 2016 Nov;18(11):1342-50. doi:10.1002/ejhf.663.

22. Jackson CE, Haig C, Welsh P, Dalzell JR, Tsorlalis IK, McConnachie A, Preiss D, Anker SD, Sattar N, Petrie MC, Gardner RS, McMurray JJ. The incremental prognostic and clinical value of multiple novel biomarkers in heart failure. Eur J Heart Fail. 2016 Dec;18(12):1491-8. doi:10.1002/ejhf.543.

23. Morishita T, Uzui H, Mitsuke Y, Amaya N, Kaseno K, Ishida K, Fukuoka Y, Ikeda H, Tama N, Yamazaki T, Lee JD, Tada H. Association between matrix metalloproteinase-9 and worsening heart failure events in patients with chronic heart failure. ESC Heart Fail. 2017 Aug;4(3):321-30. doi:10.1002/ehf2.12137.

24. Täger $T$, Wiebalck $C$, Fröhlich $H$, et al. Biological variation of extracellular matrix biomarkers in patients with stable chronic heart failure. Clin Res Cardiol. 2017;106:974-85.

25. Dörr O, Walther $C$, Liebetrau C, Keller T, Tabert H, Boeder N, Bayer M, Bauer P, Möllmann H, Gaede L, Troidl C, Voss S, Bauer T, Hamm CW, Nef H. Specific biomarkers of myocardial inflammation and remodeling processes as predictors of mortality in high-risk patients undergoing percutaneous mitral valve repair (MitraClip). Clin Cardiol. 2018 Apr;41(4):481-7. doi:10.1002/clc.22900.

26. Lan TH, Huang XQ, Tan HM. Vascular fibrosis in atherosclerosis. Cardiovasc Pathol. 2013 SepOct;22(5):401-7. doi:10.1016/j.carpath.2013.01.003.

27. Nilsson L, Hallén J, Atar D, Jonasson L, Swahn E. Early measurements of plasma matrix metalloproteinase-2 predict infarct size and ventricular dysfunction in ST-elevation myocardial infarction. Heart. 2012 Jan;98(1):31-6. doi: 10.1136/heartjnl-2011-300079.

28. Peeters SA, Engelen L, Buijs J, Jorsal A, Parving HH, Tarnow L, Rossing P, Schalkwijk CG, Stehouwer CDA. Plasma matrix metalloproteinases are associated with incident cardiovascular disease and allcause mortality in patients with type 1 diabetes: a 12-year follow-up study. Cardiovasc Diabetol. 2017 Apr 26;16(1):55. doi: 10.1186/s12933-017-0539-1.

29. Chung AW, Yang HH, Kim JM, Sigrist MK, Chum E, Gourlay WA, Levin A. Upregulation of matrix metalloproteinase- 2 in the arterial vasculature contributes to stiffening and vasomotor dysfunction in patients with chronic kidney disease. Circulation. 2009 Sep 1;120(9):792-801. doi: 10.1161/CIRCULATIONAHA.109.862565.

30. Peeters SA, Engelen L, Buijs J, Chaturvedi N, Fuller JH, Schalkwijk CG, Stehouwer CD. EURODIAB Prospective Complications Study Group. Plasma levels of matrix metalloproteinase-2, $-3,-10$, and tissue inhibitor of metalloproteinase- 1 are associated with vascular complications in patients with type 1 diabetes: the EURODIAB Prospective Complications Study. Cardiovasc Diabetol. $2015 \mathrm{Mar}$ 10;14:31. doi: 10.1186/s12933-015-0195-2. 
31. Du X, Shimizu A, Masuda Y, Kuwahara N, Arai T, Kataoka M, Uchiyama M, Kaneko T, Akimoto T, lino Y, Fukuda Y. Involvement of matrix metalloproteinase-2 in the development of renal interstitial fibrosis in mouse obstructive nephropathy. Lab Invest. 2012 Aug;92(8):1149-60.

doi:10.1038/labinvest.2012.68.

32. Yu XA, Hu Y, Zhang Y, Zhang R, Bai X, Gu L, Gao H, Li R, Tian J, Yu BY. Integrating the Polydopamine Nanosphere/Aptamers Nanoplatform with a DNase-I-Assisted Recycling Amplification Strategy for Simultaneous Detection of MMP-9 and MMP-2 during Renal Interstitial Fibrosis. ACS Sens. 2020 Apr 24;5(4):1119-1125. doi: 10.1021/acssensors.0c00058.

33. Chen NX, O'Neill KD, Chen X, Kiattisunthorn K, Gattone VH, Moe SM. Activation of arterial matrix metalloproteinases leads to vascular calcification in chronic kidney disease. Am J Nephrol. 2011;34(3):211-9. doi:10.1159/000330175.

34. Hsu T-W, Kuo K-L, Sz-Ch H, Huang P-H, Chen J-W, Tarng D-Ch. Progression of Kidney Disease in NonDiabetic Patients with Coronary Artery Disease: Predictive Role of Circulating Matrix Metalloproteinase-2, -3, and - 9. PLoS One. 2013;8(7):e70132. doi:10.1371/journal.pone.0070132.

\section{Tables}

Table 1. Detailed characteristics of the study group. 


\begin{tabular}{|c|c|c|c|c|c|c|c|}
\hline & Mean & Median & SD & Minimum & Maximum & Shapiro-Wilk & $95 \% \mathrm{CI}$ \\
\hline MMP-2 [ng/mL] & 208 & 192 & 62.97 & 133 & 446 & $<0.00001$ & $190-225$ \\
\hline MMP-9 [ng/mL] & 851 & 799 & 438 & 166 & 1946 & 0.0159 & $736-966$ \\
\hline MMP-3 [ng/mL] & 18.41 & 16.33 & 8.71 & 5.12 & 49.88 & 0.00061 & $16.06-20.76$ \\
\hline TIMP-2 [ng/mL] & 91.87 & 84.85 & 27.25 & 56.6 & 183 & 0.00006 & $84.64-99.10$ \\
\hline Age [years] & 36.76 & 33 & 13.88 & 18 & 70 & 0.00021 & $33.11-40.41$ \\
\hline $\mathrm{CRP}[\mathrm{mg} / \mathrm{L}]$ & 45.60 & 26.47 & 59.87 & 0.12 & 303 & $<0.00001$ & $29.71-61.49$ \\
\hline $\mathrm{BNP}[\mathrm{pg} / \mathrm{mL}]$ & 254 & 55.1 & 529 & 10 & 2197 & $<0.00001$ & $102-406$ \\
\hline Troponin [ng/mL] & 9.09 & 4.97 & 11.63 & 0.01 & 44.8 & $<0.00001$ & $6.77-18.36$ \\
\hline Leukocytes $\left[10^{3} / \mu \mathrm{L}\right]$ & 9.52 & 8.86 & 3.52 & 3.99 & 18.82 & 0.00081 & 8.59-10.44 \\
\hline Erythrocytes $\left[10^{6} / \mu \mathrm{L}\right]$ & 4.79 & 4.84 & 0.59 & 3.12 & 5.82 & 0.0376 & $4.64-4.95$ \\
\hline Hematocrit [\%] & 42.3 & 42.3 & 4.60 & 29.1 & 50 & 0.0259 & $41.09-43.51$ \\
\hline Hemoglobin [g/dL] & 14.4 & 14.55 & 1.81 & 9.4 & 17.4 & 0.0964 & $13.92-14.87$ \\
\hline Potassium [mmol/L] & 4.21 & 4.19 & 0.46 & 3.1 & 5.35 & 0.816 & $4.09-4.33$ \\
\hline Sodium $[\mathrm{mmol} / \mathrm{L}]$ & 139 & 139 & 2.41 & 132 & 143 & 0.0409 & $138-140$ \\
\hline Magnesium [mg/dL] & 2.15 & 2.10 & 0.23 & 1.76 & 2.7 & 0.0992 & $2.09-2.21$ \\
\hline Creatinine $[\mathrm{mg} / \mathrm{dL}]$ & 0.97 & 0.96 & 0.17 & 0.68 & 1.45 & 0.0969 & $0.92-1.01$ \\
\hline eGFR & 89.64 & 92 & 23.85 & 6 & 132 & 0.0005 & 83.37-95.91 \\
\hline LVEF [\%] & 54.29 & 60 & 14.56 & 15 & 75 & 0.00109 & $50.47-58.12$ \\
\hline LVESD [mm] & 36.5 & 35 & 8.99 & 22 & 62 & 0.00009 & $34.14-38.86$ \\
\hline LVEDD [mm] & 53.64 & 52 & 6.53 & 42 & 70 & 0.00269 & $51.92-55.36$ \\
\hline GLS [\%] & -15.52 & -16.5 & 5.56 & -24.3 & -1.1 & 0.0414 & $-17.60-(-13.44)$ \\
\hline MR LVEF [\%] & 55.85 & 59 & 13.47 & 12 & 75 & 0.00012 & $51.8-59.9$ \\
\hline MR LVESD [mm] & 38.38 & 36 & 8.02 & 29 & 64 & 0.00003 & $3.60-4.08$ \\
\hline MR LVEDD [mm] & 53.98 & 54 & 5.66 & 44 & 70 & 0.011 & $5.23-5.57$ \\
\hline
\end{tabular}

Abbreviations: 95\% CI, 95\% confidence interval for the mean; BNP, B-type natriuretic peptide; CRP, C-reactive protein; eGFR, estimated glomerular filtration rate; GLS, echocardiographic global longitudinal strain; LVEDD, left ventricular enddiastolic dimension in echocardiography; LVEF, left ventricular ejection fraction in echocardiography; LVESD, left ventricular endsystolic dimension in echocardiography; MMP, matrix metalloproteinase; MR LVEDD, left ventricular end-diastolic dimension in cardiac magnetic resonance imaging; MR LVEF, left ventricular ejection fraction in cardiac magnetic resonance imaging; MR LVESD - left ventricular end-systolic dimension in cardiac magnetic resonance imaging; SD, standard deviation; Shapiro-Wilk, Shapiro-Wilk normality test; TIMP, tissue inhibitor of metalloproteinases

Table 2. Validity of variables based on the principal component analysis (PCA). 


\begin{tabular}{|c|c|c|c|}
\hline Validity of variables & Class & Strength & Validity \\
\hline Significant heart disease YES & 1 & 0.675568 & 2 \\
\hline Significant heart disease NO & 2 & 0.675568 & 2 \\
\hline LVEF & & 0.611645 & 3 \\
\hline LVESD & & 0.598840 & 4 \\
\hline eGFR & & 0.571225 & 5 \\
\hline MMP-2 & & 0.571186 & 6 \\
\hline TIMP-2 & & 0.564665 & 7 \\
\hline ECHO contractility disorders YES & 1 & 0.480446 & 9 \\
\hline ECHO contractility disorders NO & 2 & 0.480446 & 9 \\
\hline LVEDD & & 0.476995 & 10 \\
\hline ECG Q wave NO & 1 & 0.452301 & 12 \\
\hline ECG Q wave YES & 2 & 0.452301 & 12 \\
\hline ECG ST depression NO & 1 & 0.443172 & 14 \\
\hline ECG ST depression YES & 2 & 0.443172 & 14 \\
\hline Age & & 0.409799 & 15 \\
\hline Male & 1 & 0.404991 & 17 \\
\hline Female & 2 & 0.404991 & 17 \\
\hline ECG ST elevation NO & 1 & 0.380145 & 19 \\
\hline ECG ST elevation YES & 2 & 0.380145 & 19 \\
\hline ECG inverted T wave YES & 1 & 0.299024 & 21 \\
\hline ECG inverted T wave NO & 2 & 0.299024 & 21 \\
\hline MMP-3 & & 0.258577 & 22 \\
\hline ECG BBB NO & 1 & 0.120286 & 24 \\
\hline ECG BBB YES & 2 & 0.120286 & 24 \\
\hline MMP-9 & & 0.103218 & 25 \\
\hline Creatinine & & 0.096657 & 26 \\
\hline
\end{tabular}

Abbreviations: BBB, bundle branch block (right or left); eGFR, estimated glomerular filtration rate; LVEDD, left ventricular enddiastolic dimension in echocardiography; LVEF, left ventricular ejection fraction in echocardiography; LVESD, left ventricular endsystolic dimension in echocardiography; MMP, matrix metalloproteinase; TIMP, tissue inhibitor of metalloproteinases

Table 3. Univariate logistic regression analysis for a significant history of heart disease. 


\begin{tabular}{|l|c|c|c|}
\hline \multicolumn{1}{|c|}{ Variable } & Odds ratio & $95 \%$ CI & $\mathrm{p}$ \\
\hline MMP-2 & 1.020 & $1.006-1.034$ & 0.004 \\
\hline MMP-3 & 1.092 & $1.009-1.182$ & 0.025 \\
\hline TIMP-2 & 1.048 & $1.017-1.081$ & 0.002 \\
\hline Age & 1.120 & $1.053-1.192$ & $<0.001$ \\
\hline eGFR & 1.087 & $1.029-1.149$ & 0.002 \\
\hline LVEF & 1.089 & $1.034-1.147$ & $<0.001$ \\
\hline LVESD & 1.176 & $1.070-1.293$ & $<0.001$ \\
\hline LVEDD & 1.213 & $1.077-1.366$ & 0.001 \\
\hline GLS & 1.505 & $1.091-2.076$ & 0.009 \\
\hline MR LVEF & 1.093 & $1.022-1.169$ & 0.008 \\
\hline MR LVESD & 4.573 & $1.519-13.766$ & 0.005 \\
\hline MR LVEDD & 7.194 & $1.483-34.902$ & 0.012 \\
\hline Female sex & 11.143 & $2.627-47.264$ & 0.001 \\
\hline Segmental contractility disorders & 12.133 & $2.412-61.031$ & 0.002 \\
\hline
\end{tabular}

Abbreviations: 95\% CI, 95\% confidence interval; eGFR, estimated glomerular filtration rate; GLS, echocardiographic global longitudinal strain; LVEDD, left ventricular end-diastolic dimension in echocardiography; LVEF, left ventricular ejection fraction in echocardiography; LVESD, left ventricular end-systolic dimension in echocardiography; MMP, matrix metalloproteinase; MR LVEDD, left ventricular end-diastolic dimension in cardiac magnetic resonance imaging; MR LVEF, left ventricular ejection fraction in cardiac magnetic resonance imaging; MR LVESD - left ventricular end-systolic dimension in cardiac magnetic resonance imaging; TIMP, tissue inhibitor of metalloproteinases

Table 4. Correlation of creatinine, eGFR and matrix metalloproteinases levels with tissue inhibitor level.

\begin{tabular}{|c|c|c|}
\hline & Creatinine & eGFR \\
\hline \multirow{2}{*}{ MMP-2 } & 0.156 & -0.535 \\
\cline { 2 - 3 } & $\mathrm{p}=0.269$ & $p<0.001$ \\
\hline \multirow{2}{*}{ MMP-9 } & 0.083 & -0.102 \\
\cline { 2 - 3 } & $\mathrm{p}=0.560$ & $\mathrm{p}=0.470$ \\
\hline \multirow{2}{*}{ MMP-3 } & 0.464 & -0.439 \\
\cline { 2 - 3 } & $p=0.001$ & $p=0.001$ \\
\hline \multirow{2}{*}{ TIMP-2 } & 0.249 & -0.564 \\
\cline { 2 - 3 } & $\mathrm{p}=0.075$ & $p<0.001$ \\
\hline
\end{tabular}

Abbreviations: eGFR, estimated glomerular filtration rate; MMP, matrix metalloproteinase; TIMP, tissue inhibitor of metalloproteinases

Table 5. Correlation of biochemical values with the results of imaging studies in echocardiography and magnetic resonance imaging. 


\begin{tabular}{|c|c|c|c|c|c|c|c|}
\hline & LVEF & LVESD & LVEDD & GLS & MR LVEF & MR LVESD & MR LVEDD \\
\hline \multirow{2}{*}{ MMP-2 } & -0.555 & 0.539 & 0.229 & 0.407 & -0.373 & 0.338 & 0.216 \\
\cline { 2 - 8 } & $p=0.007$ & $p=0.010$ & $\mathrm{p}=0.306$ & $\mathrm{p}=0.060$ & $\mathrm{p}=0.087$ & $\mathrm{p}=0.124$ & $\mathrm{p}=0.334$ \\
\hline \multirow{2}{*}{ MMP-9 } & -0.218 & 0.308 & 0.196 & 0.253 & -0.084 & 0.220 & 0.044 \\
\cline { 2 - 8 } & $\mathrm{p}=0.329$ & $\mathrm{p}=0.164$ & $\mathrm{p}=0.381$ & $\mathrm{p}=0.257$ & $\mathrm{p}=0.711$ & $\mathrm{p}=0.325$ & $\mathrm{p}=0.845$ \\
\hline \multirow{2}{*}{ MMP-3 } & -0.517 & 0.482 & 0.299 & 0.571 & -0.319 & 0.324 & 0.284 \\
\cline { 2 - 8 } & $p=0.014$ & $p=0.023$ & $\mathrm{p}=0.177$ & $p=0.006$ & $\mathrm{p}=0.147$ & $\mathrm{p}=0.141$ & $\mathrm{p}=0.201$ \\
\hline \multirow{2}{*}{ TIMP-2 } & -0.685 & 0.668 & 0.426 & 0.540 & -0.443 & 0.435 & 0.294 \\
\cline { 2 - 8 } & $p<0.001$ & $p=0.001$ & $p=0.048$ & $p=0.010$ & $p=0.039$ & $p=0.043$ & $\mathrm{p}=0.184$ \\
\hline
\end{tabular}

Abbreviations: GLS, echocardiographic global longitudinal strain; LVEDD, left ventricular end-diastolic dimension in echocardiography; LVEF, left ventricular ejection fraction in echocardiography; LVESD, left ventricular end-systolic dimension in echocardiography; MMP, matrix metalloproteinase; MR LVEDD, left ventricular end-diastolic dimension in cardiac magnetic resonance imaging; MR LVEF, left ventricular ejection fraction in cardiac magnetic resonance imaging; MR LVESD - left ventricular end-systolic dimension in cardiac magnetic resonance imaging; TIMP, tissue inhibitor of metalloproteinases

Figures

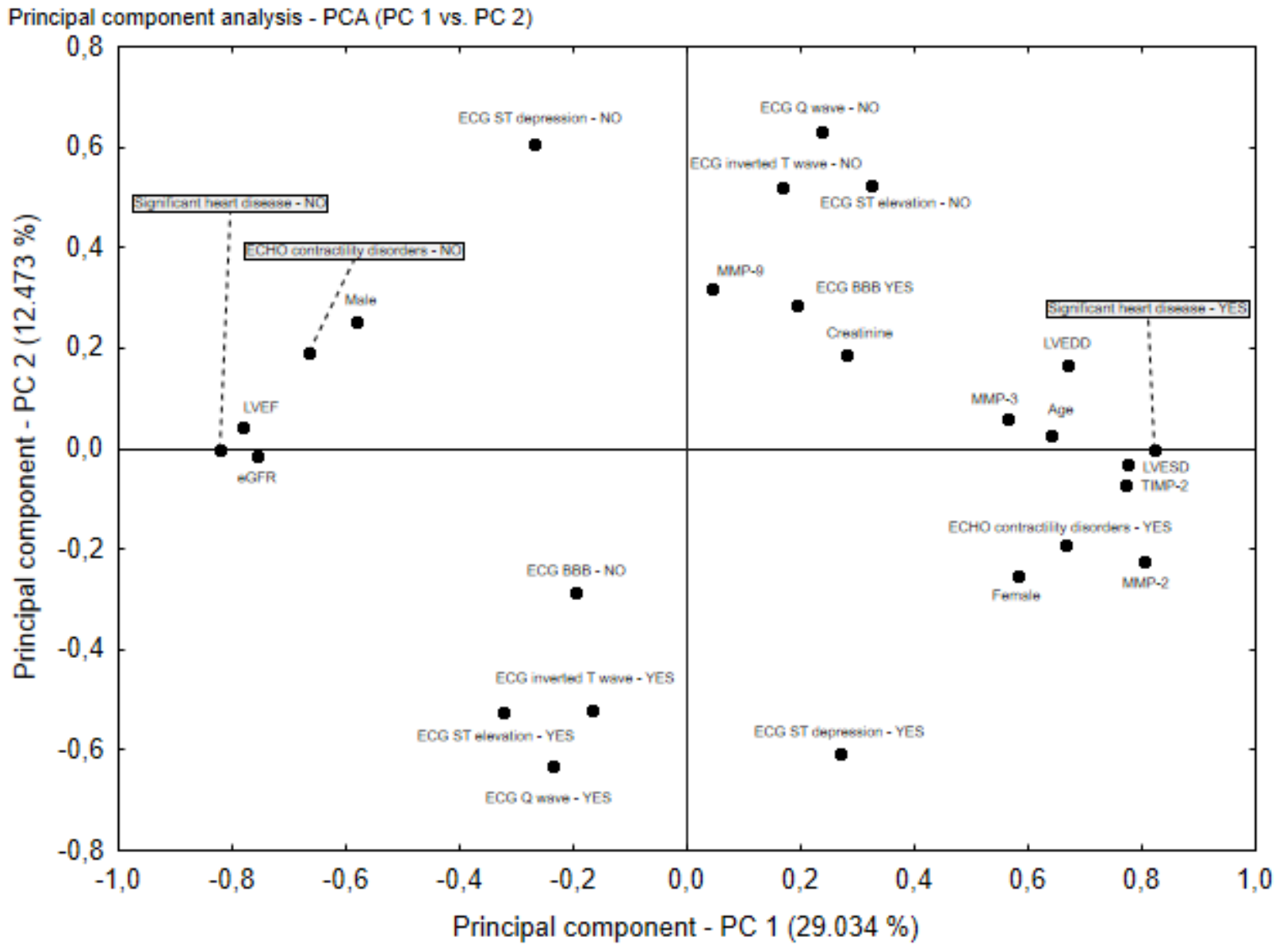

Figure 1 
Principal component analysis (PCA). Abbreviations: BBB, bundle branch block (right or left); eGFR, estimated glomerular filtration rate; LVEDD, left ventricular end-diastolic dimension in echocardiography; LVEF, left ventricular ejection fraction in echocardiography; LVESD, left ventricular end-systolic dimension in echocardiography; MMP, matrix metalloproteinase; TIMP, tissue inhibitor of metalloproteinases

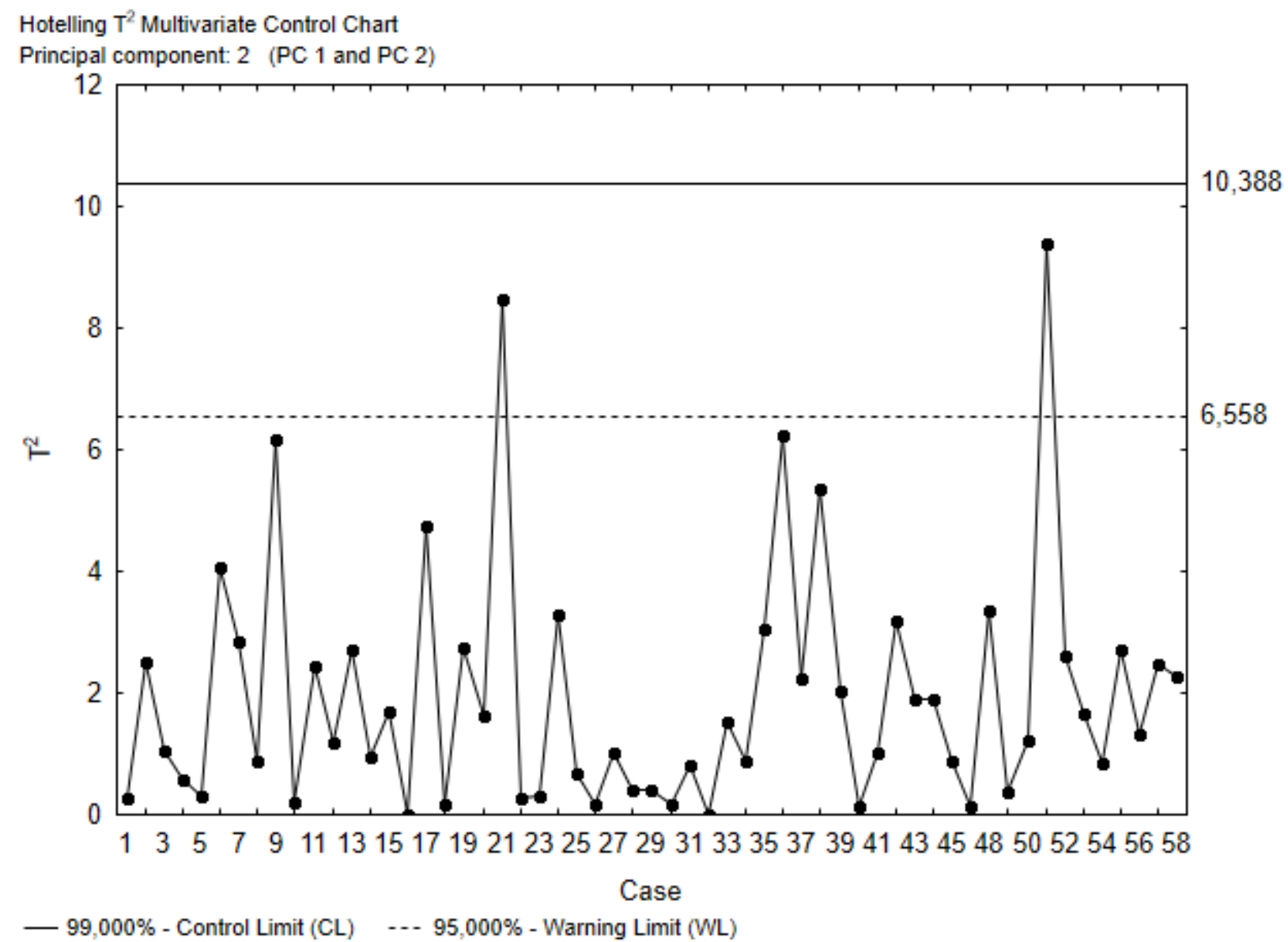

Figure 2

Hotelling T2 Multivariate Control Chart 